\title{
Cyclosporin A inhibits the growth of neonatal MHC-expressing myotubes independent of NFATc1 and NFATc3 in the mechanically overloaded soleus muscle of mice
}

\author{
Kunihiro Sakuma ${ }^{1}$, Akihiko Yamaguchi ${ }^{2}$ \\ ${ }^{1}$ Research Center for Physical Fitness, Sports and Health, Toyohashi University of Technology, Toyohashi, Japan; \\ ${ }^{2}$ School of Dentistry, Health Sciences University of Hokkaido, Hokkaido, Japan. \\ Email: ksakuma@las.tut.ac.jp, yama@hoku-iryo-u.ac.jp
}

Received 2 March 2011; revised 29 March 2011; accepted 6 April 2011.

\section{ABSTRACT}

The molecular signaling pathway linked to hypertrophy of the anti-gravity/postural soleus muscle after mechanical overloading has not been identified. Using Western blot and immunohistochemical analyses, we investigated whether the amounts of NFATc3, GSK-3 $\beta$, NFATc1, and neonatal MHC change in the mechanically overloaded soleus muscle after cyclosporin A (CsA) treatment. Adult male ICR mice were subjected to a surgical ablation of the gastrocnemius muscle and treated with either CsA (25 $\mathrm{mg} / \mathrm{Kg}$ ) or vehicle once daily. They were sacrificed at $2,4,7,10$, and 14 days post-injury. Mechanical overloading resulted in a significant increase in the wet weight and the cross-sectional area of slow and fast fibers of the soleus muscle in placebo-treated mice but not CsA-treated mice. After 4 days of mechanical overloading, we observed a similar co-localization of neonatal MHC and NFATc3 in several myotubes of both mice. The placebo-treated mice possessed larger myotubes with neonatal MHC than CsA-treated mice. At 7 days, mechanical overloading induced marked expression of neonatal MHC in myotubes and/or myofibers. Such neonatal MHC-positive fibers emerged less often in the hypertrophied soleus muscle subjected to treatment with CsA. CsA treatment did not significantly change the amount of GSK-3 $\beta$ protein in the soleus muscle. The modulation of growth in neonatal MHC-positive myofibers by CsA treatment may inhibit the hypertrophic process in the soleus muscle after mechanical overloading.

Keywords: Calcineurin; NFATc3; Neonatal MHC; Skeletal Muscle; Hypertrophy

\section{INTRODUCTION}

Skeletal muscle mass is increased in response to positive changes in workload or activity as a result of hypertrophy of individual muscle fibers, but the key molecular mediators of hypertrophy are only beginning to be elucidated [1]. Induction of hypertrophy in adult skeletal muscle is accompanied by the increased expression of insulin-like growth factor 1 (IGF-1) [2,3]. In fact, several researchers clearly showed that the mRNA level and immunoreactivity of IGF-I were markedly increased in the hypertrophied plantaris muscle by surgical ablation of co-operative muscles [2,3]. When IGF-1 levels were enhanced by using a muscle-specific promoter in transgenic mice, increased muscle size resulted $[4,5]$. These lines of evidence support the idea that the hypertrophy of mature skeletal muscle can be mediated by pathways activated by autocrine or paracrine sources of IGF-1.

The binding of IGF-1 to its receptor triggers the activation of several intracellular kinases, including phosphatidylinositol-3-kinase (PI3K). PI3K phosphorylates the membrane phospholipid phosphatidylinositol-4, 5-bisphosphate, creating a lipid-binding site on the cell membrane for a serine/threonine kinase called Akt (also called Akt1 or protein kinase B). Fiber hypertrophy of the plantaris muscle after mechanical overloading was completely blocked by treatment with rapamycin, an inhibitor for the downstream molecule Akt, the mammalian target of rapamycin (mTOR) [6]. In addition, a transgenic mouse with a constitutively active form of Akt in adult skeletal muscle exhibited rapid and significant skeletal muscle hypertrophy [7].

Another target stimulated by IGF-I signaling is calcineurin. $\mathrm{A} \mathrm{Ca}^{2+} /$ calmodulin-dependent protein serine/threonine phosphatase, calcineurin has been implicated as a molecular decoder modulating the differentiated myoblasts that fuse into myotubes [8] and regenera- 
tion of skeletal muscle fibers [9-13] or enhancing the slower transformation of myosin heavy chain (MHC) and fiber types [14-16]. Although several lines of evidence exclude a functional role for calcineurin in the hypertrophy of muscle fibers in vivo $[6,16,17]$, much evidence obtained using several transgenic mice or pharmacological inhibition of calcineurin [13,18,19], indicates a selective influence of calcineurin on the size of the anti-gravity/postural soleus muscle. However, no downstream modulator of calcineurin signaling has been identified.

Interestingly, immature myotubes expressing developmental MHC exist in the mechanically overloaded muscle [15,20-22], although it is believed blindly that muscle hypertrophy does not include the neo-formation of muscle fibers [23]. Several previous findings [24,25] imply a break in the integrity of the plasma membrane to be essential for muscle hypertrophy, releasing chemical mediators (growth factors) of remodeling stored in the cell cytosol (e.g. FGF). If this-is the case, the muscle hypertrophy elicited by mechanical stress would include a damage-regeneration (remodeling) phase, during which immature myotubes would emerge. In fact, mechanical overloading of skeletal muscle leads to wide-ranging inflammation [26,27] and the expression of inflammatory cytokine in immune cells [28]. Furthermore, the growth of myotubes seems to be regulated by NFATc1 and/or NFATc3, downstream targets of calcineurin signaling. After stimulation by IGF-I and calmodulin, calcineurin promoted myotube growth by co-operating with NFATc1 and GATA-2 [29]. On the other hand, overexpression of calcineurin and NFATc3 dramatically enhanced the differentiation and expression of slow MHC in 10T1/2 cells in vitro [30]. These results prompt us to hypothesize that NFATc1 and/or NFATc3 by stimulating calcineurin enhance the growth of immature fibers in the soleus muscle after mechanical overloading.

The purpose of this study was twofold. The first goal was to determine whether the fiber hypertrophy of mature soleus muscle includes neonatal MHC-positive myotubes. The second was to determine whether the pharmacological inhibition of calcineurin activity modulates the expression pattern of neonatal MHC, NFATc1 and NFATc3 in the overloaded soleus muscle in vivo.

\section{MATERIALS AND METHODS}

\subsection{Experimental Animals}

Ninety-two male ICR mice (30 - 40g, 10 - 12 weeks) were used in the experiments. The mice were housed in a temperature $(22 \pm 2)$ and humidity (60 \pm $5 \%$ )-controlled room regulated to provide alternating 12-h periods of light and darkness. They were allowed to feed (commercial rat chow) and drink ad libitum. This experimental procedure was approved by the Committee for Animal Research in Toyohashi University of Technology.

\subsection{Compensatory Enlargement and Cyclosporin A Injection}

Compensatory enlargement of the soleus muscle was induced in one leg of each mouse by surgical removal of the ipsilateral gastrocnemius muscles as described previously [18]. A sham operation was performed on the contralateral leg after the mice were anesthetized with an intraperitoneal injection of sodium pentobarbital. These mice were treated with either CsA $(25 \mathrm{mg} / \mathrm{kg}$, intraperitoneally, $n=30$ ) or vehicle (10\% Cremophor (Novartis) and $5 \%$ Ethanol, $n=30$ ) once daily. Treatment with these chemical agents did not affect health (from general observations or autopsy results), growth (body weight did not differ among mice at any time during the treatment), or the daily amount of locomotive activity displayed by experimental animals. Using an excess pentobarbital administration, mice were killed and the soleus muscles of both legs were dissected at $2(N=12)$, $4(n=28), 7(N=12), 10(n=28)$, and $14(N=12)$ days post-surgery.

\subsection{Primary Antibodies}

The antibodies employed in the present study were as follows: affinity-purified mouse monoclonal antibody to neonatal MHC (1 : 50, Cat. No. MONX10808, SANBIO B.V., The Netherlands), affinity-purified rabbit polyclonal antibody to NFATc3 (1 : 100, Cat. No. M-225, Santa Cruz Biotechnology Inc., Santa Cruz, CA, USA), to GSK3 $\beta$ (1 : 1000, Cat. No. \#9315, Cell signaling, Beverly, MA, USA) and to P-GSK-3 $\beta$ [1 : 1000, Cat. No. \#9293, Cell signaling], and affinity-purified goat polyclonal antibody to actin and to NFATc1 (Santa Cruz Biotechnology). The NFATc3 antibody was raised against an epitope mapping near the C-terminus of NFATc3 of human origin. This antibody recognizes the NFATc3 protein and did not cross-react with the other members of the NFAT family (NFATc1, NFATc2 and NFATc4).

\subsection{Tissue Preparation, Gel Electrophoresis, and Immunoblotting}

The soleus muscle of both legs at 4 and 10 days (each timepoint, placebo-treated, $n=8$; CsA-treated, $n=8$; total $n=32$ ) postsurgery was homogenized in 10-20 vols of $50 \mathrm{mM}$ Tris-HCl pH 7.4, $5 \mathrm{mM}$ EDTA, $10 \mu \mathrm{g} / \mathrm{ml}$ phenylmethylsulfonylfluoride, $0.5 \mu \mathrm{g} / \mathrm{ml}$ leupeptin, 0.2 $\mu \mathrm{g} / \mathrm{ml}$ aprotinin, $0.2 \% \mathrm{NP}-40,0.1 \%$ Triton X-100, and $1 \mathrm{mM} \mathrm{Na} \mathrm{VO}_{4}$ in a polytron (DIAX 900, Hei- 
dolph-Instruments, Schwabach, Germany) for $30 \mathrm{~s}$. The homogenized tissues were then centrifuged at $15,000 \times \mathrm{g}$ for $25 \mathrm{~min}$ at $4^{\circ} \mathrm{C}$, and the protein concentration of the supernatant was colorimetrically determined (Bio-Rad protein determination kit, Bio-Rad Laboratories, Richmond, CA). Sodium dodecylsulfate-poly- acrylamide gel electrophoresis (SDS-PAGE) (12.5\% acrylamide) and Western blotting were performed next as described previously [18]. Proteins separated by SDS-PAGE were electrophoretically transferred onto nitrocellulose membranes (Hybond-ECL Western, Amersham, Arlington Heights, IL). The blots were then incubated with blocking buffer composed of $0.1 \%$ Tween- 20 and $1 \%$ gelatin in $10 \mathrm{mM}$ Tris-buffered saline (TBS, $10 \mathrm{mM}$ Tris, 135 $\mathrm{mM} \mathrm{NaCl}, 1 \mathrm{mM} \mathrm{KCl}$, and $0.02 \% \mathrm{NaN}_{3}$, pH 7.4) for 10 min. The blots were next incubated with primary antibodies overnight, and with goat anti-rabbit IgG-conjugated AP (1 : 10000, Promega Co., Madison, USA) for lh and were visualized with Western blue, a stabilized substrate for alkaline phosphatase (Promega). A densitometric analysis of each blot was performed with a computerized image processing system (NIH Image 1.63).

\subsection{Immunofluorescence}

Mouse soleus muscle was isolated and frozen in isopentane at 2, 4, 7 and 10 (each timepoint, placebo-treated, $n$ = 6; CsA-treated, $n=6$; total $n=48$ ) days postsurgery. Serial $7-\mu \mathrm{m}$ transverse sections made with a cryostat (Microm Coldtome, HM-520, Germany) were mounted on silanized slides (Dako Japan, Tokyo). Cryosections were fixed with methanol (3 $\mathrm{min})$ and incubated in blocking solution $(2.5 \%-10 \%$ normal horse serum in PBS) for $30 \mathrm{~min}$ at room temperature. Sections were incubated for 60 min with anti- NFATc3 or NFATc1 and anti-neonatal $\mathrm{MHC}$ antibodies at $4^{\circ} \mathrm{C}$ overnight. After being washed in PBS, sections were incubated with anti-mouse or anti-rabbit FITC-conjugated (1:100 final dilution; Rockland Immunochemicals, Inc., USA) and anti-goat Rhodamine-conjugated (1 : 100 final dilution; Chemicon International Inc., USA) secondary antibodies. The muscle sections were mounted in a slowfade antifade kit with 4'-6-Diamidino-2-phenylindole (DAPI) (Molecular Probes, Eugene, OR). Images were acquired on an Olympus BX50 inverted microscope with a fluorescent attachment (Olympus) and Photonic Science CCD camera (Olympus DP70). As described previously [26], we calculated in percentages of the co-localization between neonatal MHC and NFATc3 in myotubes/ myofibers in the placebo-mechanical overloaded (MOV) and CsA-MOV mice at 2, 4, 7 and 10 days by analyzing 500 - 700 myotubes/fibers from 6-10 different parts of cross-sections using 6 animals.

\subsection{Morphometric Analysis}

At 14 days, the cross-sectional area was determined for at least 200 fibers of each type (slow and fast) at different positions throughout the muscle with an image analysis computer program (NIH Image 1.63). At 4 and 7 days, we determined the mean cross-sectional area of neonatal MHC-positive myotubes/myofibers.

\subsection{Statistical Analysis}

All values were expressed as the mean \pm S.E.M. A two-way analysis of variance (ANOVA) was used to evaluate the significance of differences in the protein levels of GSK-3 $\beta$, or the weight and cross-sectional area between two groups (placebo vs. CsA) and two conditions (sham vs. overload). Tukey's post hoc tests were conducted if the ANOVA indicated significant interactions. $\mathrm{P}<0.05$ was considered to be statistically significant.

\section{RESULTS}

To analyze the effects of CsA on the hypertrophic response of soleus muscle fibers, ICR mice were subjected to a surgical ablation of the ipsilateral gastrocnemius muscle and treated with placebo or CsA at $25 \mathrm{mg} / \mathrm{Kg}$ for 2 weeks.

The weight of the soleus muscle had significantly increased after mechanical overloading in the placebo-treated mice (control $10.8 \pm 0.3 \mathrm{mg}$ vs overload $14.6 \pm 0.8 \mathrm{mg}$ ) but not CsA-treated mice (control; $10.0 \pm$ $0.4 \mathrm{mg}$ vs overload; $11.5 \pm 1.0 \mathrm{mg}$ ). Compared with that in the non-operated soleus muscle, the cross-sectional area of slow and fast fibers was significantly increased in the mechanically overloaded soleus muscle of placebo-treated mice, by 30\% and 35\%, respectively (Table 1). The treatment with CsA had completely blocked the hypertrophy of both muscle fibers.

To understand the cellular localization of NFATc3 and neonatal MHC in mechanically overloaded soleus muscle, we performed immunofluorescence labeling of a single cryosection of the mice treated with placebo and CsA. After 2 days of mechanical overloading, immunoreactivity for NFATc3 and neonatal MHC existed in some myotubes in the soleus muscle (data not shown).

The inhibition of calcineurin did not change the expression pattern of either protein (Table 2). Figure 1 indicates the results of the quantitative analysis of NFATc3- and neonatal MHC-positive myotubes in the mechanically overloaded soleus muscle of both placeboand CsA-treated mice at 4 days. At 4 days, placebo-treated mice possessed similar amounts of neonatal MHC-positive myotubes in the muscle to CsA-treated mice. We observed a similar co-localization of neonatal MHC and NFATc3 in the soleus muscle both in pla- 

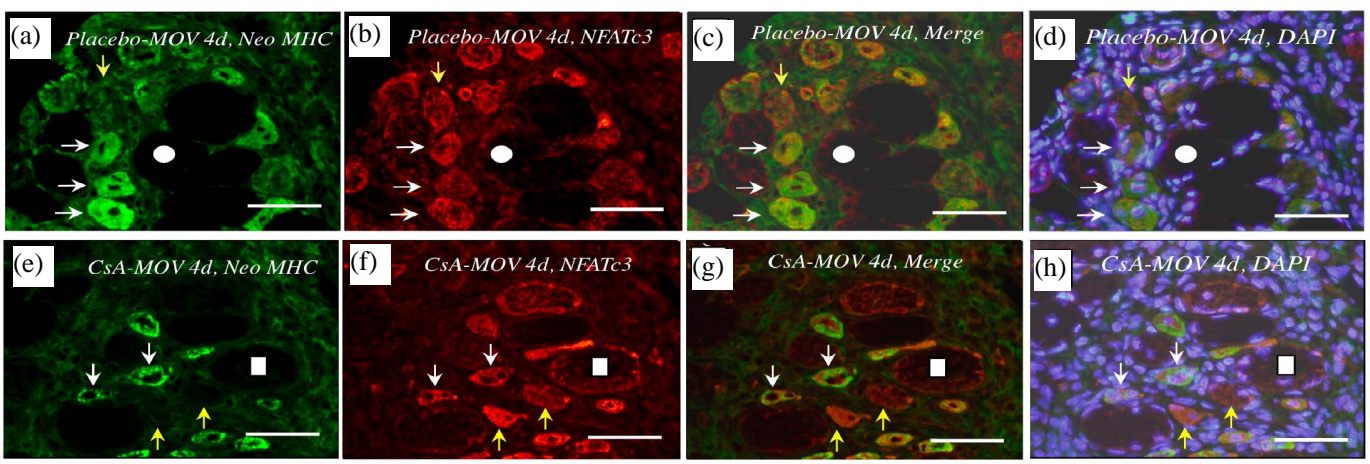

(i)

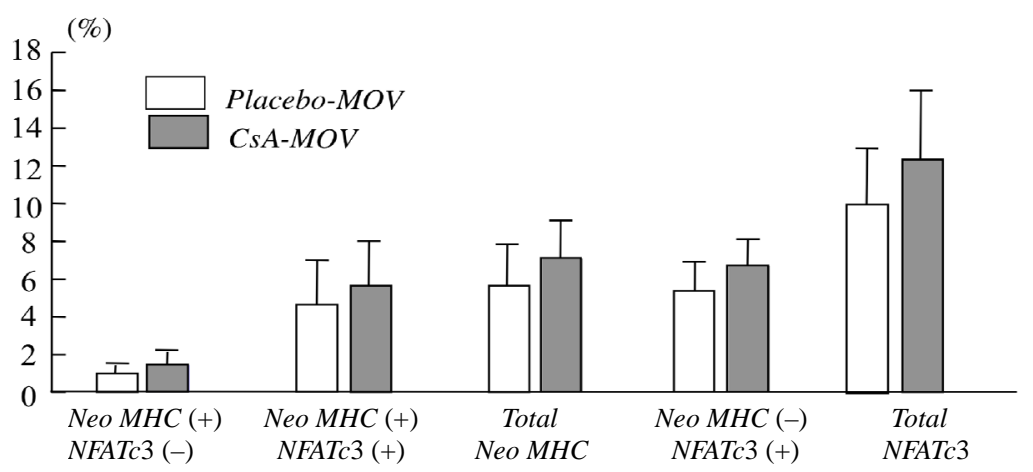

Figure 1. Cryosections of mechanically overloaded soleus muscles in mice treated with placebo ((a)-(d)) and CsA ((e)-(h)). Each cryosection was immunohistochemically stained with neonatal MHC ((a) and (e)), NFATc3 ((b) and (f)), Merge ((c) and (g)) or DAPI ((d) and (h)). At 4 days, neonatal MHC and NFATc3 were detected in cell cytosol of several immature myotubes and/or myofibers ((a) and (b)) in the overloaded soleus muscle. Calcineurin's inhibition did not change the co-localizing pattern of neonatal MHC and NFATc3 in the immature cells ((e) and (f)). The soleus muscle of CsA-treated mice appeared to have smaller neonatal MHC-positive myotubes (e). The quantitative analysis $(n=6)$ showed no significant difference between placebo-treated and CsA-treated mice on the percentages of myotubes possessing either neonatal MHC or NFATc3 (i). Neonatal MHC ((a) and (e)) and NFATc3 ((b) and (f)) immunoreactivity was visualized with FITC-and Rhodamine-conjugated secondary antibodies,respectively. White circles and squares indicate the same fibers on different immunoimages. White arrows denote the myotubes possessing both neonatal MHC and NFATc3 immunoreactivity.Yellow arrows denote the myotubes possessing NFATc3 but not neonatal MHC. Bar $=50 \mu \mathrm{m}$.

Table 1. Mean fiber area in the soleus subjected to mechanical overloading between placebo-treated and CsA-treated mice.

\begin{tabular}{llll}
\hline placebo & & CsA & \\
\hline Control & MOV 2w & Control & MOV 2w \\
Slow fiber & & Slow fiber & \\
$1456 \pm 106 \mu \mathrm{m}^{2}$ & $1914 \pm 107 \mu \mathrm{m}^{2}$ & $1510 \pm 92 \mu \mathrm{m}^{2}$ & $1396 \pm 108 \mu \mathrm{m}^{2}$ \\
Fast fiber & & Fast fiber & \\
$1439 \pm 86 \mu \mathrm{m}^{2}$ & $1829 \pm 47 \mu \mathrm{m}^{2}$ & $1279 \pm 109 \mu \mathrm{m}^{2}$ & $1369 \pm 76 \mu \mathrm{m}^{2}$ \\
\hline
\end{tabular}

cebo-treated and in CsA-treated mice. However, the soleus muscle of CsA-treated mice appeared to have smaller neonatal MHC-positive myotubes.

The co-localization pattern for NFATc3 and neonatal MHC at 7 days is demonstrated in Figure 2. Placebo-treated mice more frequently had neonatal MHC in the soleus muscle after mechanical overloading than did CsA-treated mice. Many large myotubes/ myofibers expressing neonatal MHC were observed in the placebo-treated mice at 7 days. Quantitative analysis clearly showed significant percentages of the myotubes/ myofibers to possess neonatal MHC, or both neonatal MHC and NFATc3, but not NFATc3 alone (Figure 2(i)). 

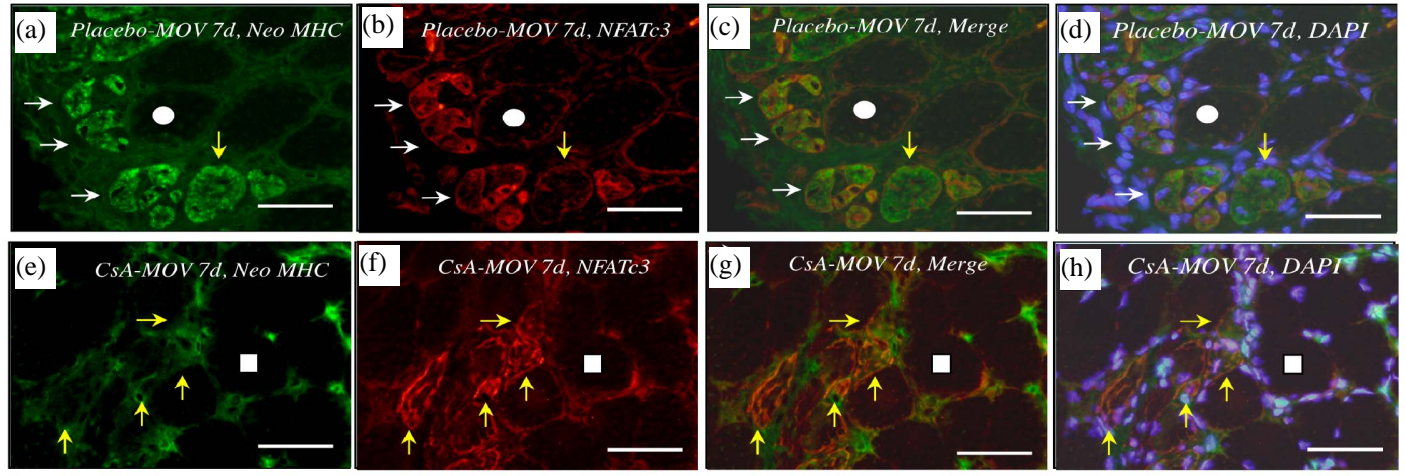

(i)

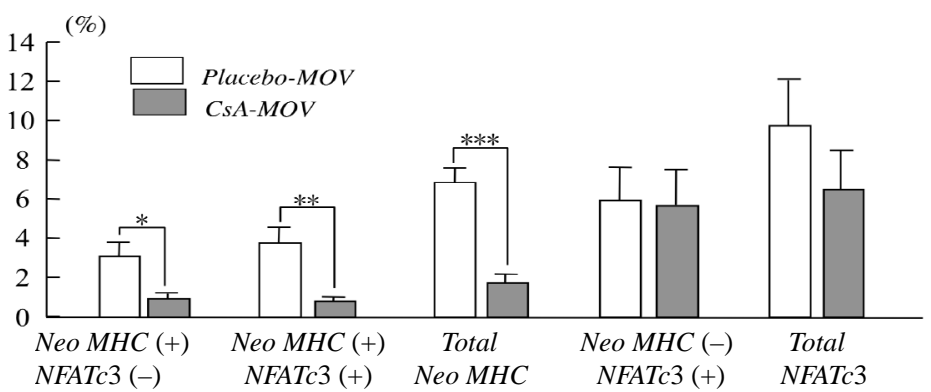

Figure 2. At 7 days post-surgery, mechanical overloading induces marked expression of both neonatal MHC and NFATc3 in a group of myotubes and/or myofibers ((a), (b) and (c)). These neonatal MHC-positive myotubes/ myofibers were very large ((a) and (c)). Calcineurin's inhibition markedly decreased the expression of neonatal MHC in the myotubes/myofibers((e), (f) and (g)). Quantitative analysis clearly showed significant increase in the percentages of the myotubes/myofibers to possess squares indicate the same fibers on different immunoimages. White arrows denote the myotubes/myofibers possessing both neonatal MHC and NFATc3 immunoreactivity. Yellow arrows denote the myotubes possessing NFATc3 but not neonatal MHC. Bar $=50 \mu \mathrm{m}$.

We examined the cross-sectional area of neonatal MHC-positive myotubes in the soleus muscle at 4 and 7 days. At 4 days, mean fiber area was significantly smaller in the CsA-treated mice than placebo-treated mice (Figure 3(a)). CsA-treated mice possessed small neonatal MHC-positive myotubes more frequently than placebo-treated mice (Figure 3(b)). The cross-sectional area of neonatal MHC-positive myotubes was significantly increased in placebo-treated mice from 4 to 7 days (Figure 3(a) and (c)).

NFATc3 is known to be regulated by GSK-3 $\beta$ [31]. More GSK-3 $\beta$ would affect the functional role of NFATc3, even if a similar amount of NFATc3 existed in the mechanically overloaded soleus muscle in both placebo-treated and CsA-treated mice. Western blot analysis showed a prominent band of GSK-3 $\beta$ at $46 \mathrm{kDa}$.

The densitometric analysis at 4 and 10 days showed no significant changes in the amount of p-GSK-3 $\beta /$ GSK-3 $\beta$ protein after mechanical overloading or CsA treatment (Figure 4).

After 10 days of mechanical overloading, immunoreactitivity for NFATc3 and neonatal MHC existed in some myotubes in the soleus muscle (data not shown). The inhibition of calcineurin did not change the expression pattern of either protein at this stage (Table 2).

We performed immunofluorescence microscopy for NFATc1 and neonatal MHC in mechanically overloaded soleus muscle. Although NFATc1- immunoreactivity was observed in some mononuclear cells, the immunolocalization of NFATc1 did not correspond that in neonatal MHC-positive myotubes (Figure 5).

\section{Discussion}

Two main conclusions can be derived from the findings of the present study. First, CsA, a potent inhibitor of calcineurin, clearly blocked the hypertrophy of fibers in the soleus muscle after surgical removal of the gastrocnemius muscle. Second, CsA at $25 \mathrm{mg} / \mathrm{Kg}$ inhibits the growth of neonatal MHC-positive myotubes and/or myofibers in the hypertrophied soleus muscle of mice.

Michel et al. found that CsA regimens using lower dosages (i.e. 5 - $10 \mathrm{mg} / \mathrm{Kg}$ ) and frequencies (once daily) of drug administration resulted in less-marked inhibition of calcineurin activity (i.e. $\leq 20 \%$ ). In fact, these lower doses of CsA failed to prevent fiber hypertrophy in overloaded rat skeletal muscles [6,32]. We selected a dosage of $25 \mathrm{mg} / \mathrm{Kg}$, because our previous studies [11,12] 

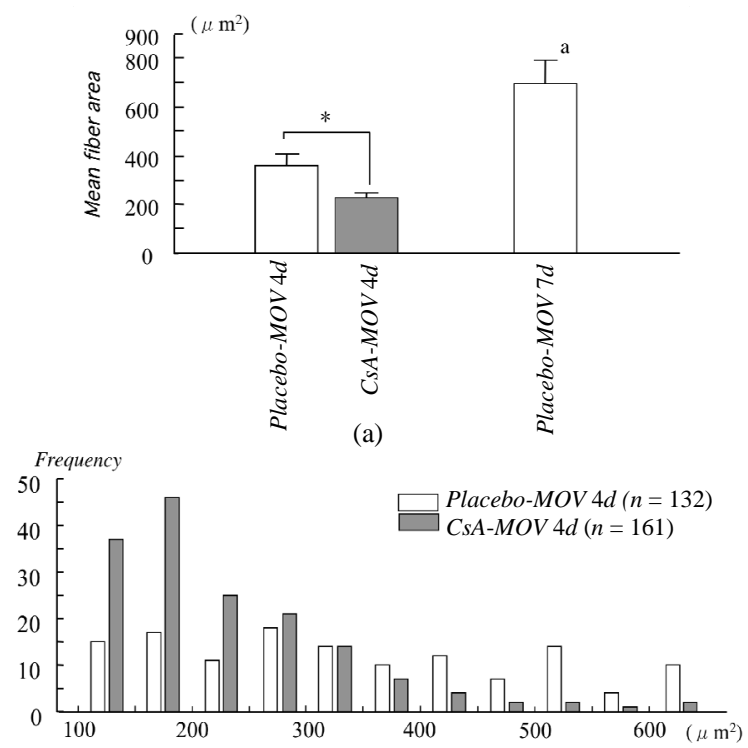

(b)

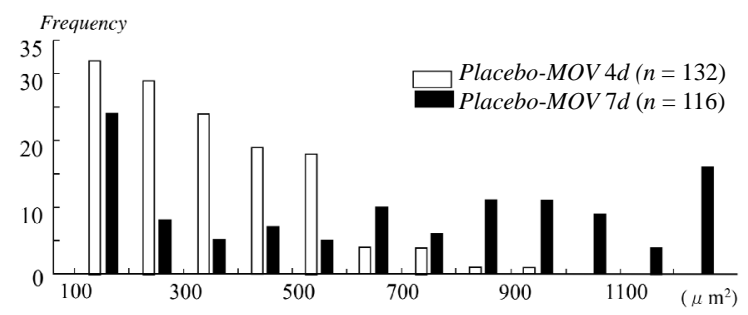

(c)

Figure 3. The cross-sectional area of neonatal MHC-positive myotubes in the soleus muscle at 4 and 7 days. At 4 days, mean fiber area was significantly smaller in the CsA-treated mice than placebo-treated mice (a). CsA- treated mice possessed small neonatal MHC-positive myotubes more frequently than placebo-treated mice (b). The cross-sectional area of neonatal MHC-positive myotubes was significantly increased in placebo-treated mice from 4 to 7 days ((a) and (c)). Values are means \pm SEM $\left(n=6\right.$ /group) $*^{*}$ and a denote significant differences with the CsA-MOV and placebo-MOV at 4 days, respectively.

demonstrated that this markedly prevents the regeneration of fibers in the tibialis anterior muscle. This dose given to mice or rats reduces calcineurin activity in skeletal muscles by $\geq 50 \%$ [32], although the present study did not examine calcineurin activity. In fact, CsA at $25 \mathrm{mg} / \mathrm{Kg}$ once daily in our previous study [18] blocked both fiber transformation and hypertrophy of the soleus muscle of mice after mechanical overloading. Therefore, the amount and frequency of CsA treatment employed here would be appropriate.

Using mechanical overloading for the plantaris muscle of rats, Dunn et al. [15] showed the appearance of immature myotubes possessing embryonic MHC. They indicated that these many embryonic myotubes regenerated fibers in the hypertrophic plantaris muscle after me-

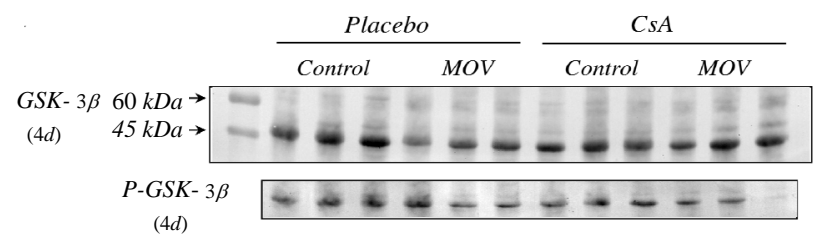

(a)

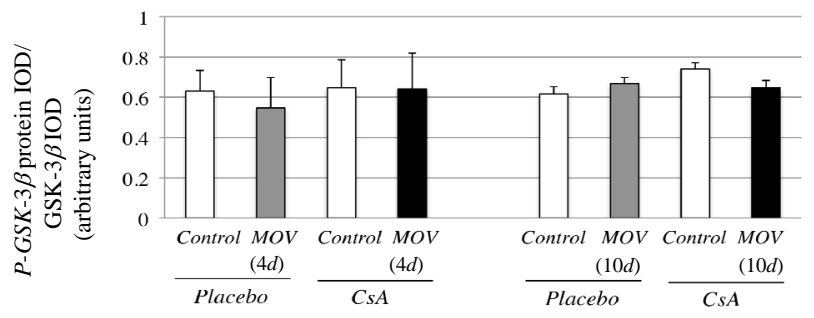

(b)

Figure 4. Pharmacological calcineurin inhibition did not modulate the amount of $\mathrm{p}-\mathrm{GSK}-3 \beta$ protein expression in the soleus muscle after MOV. (a) Western blot analysis of p-GSK- $3 \beta$ and GSK- $3 \beta$ proteins in mechanically overloaded muscle in both groups. (b) Densitometric analysis of p-GSK- $3 \beta$ and GSK- $3 \beta$ proteins in soleus muscle at 4 and 10 days after mechanical overloading. The integrated optical density (IOD) of $\mathrm{p}-\mathrm{GSK}-3 \beta$ protein normalized to the IOD of GSK- $3 \beta$ protein (arbitrary units). No significant change in level of p-GSK- $3 \beta$ and GSK- $3 \beta$ protein was observed in the soleus muscle after MOV or the treatment with CsA for 4 and 10 days. Values are means \pm SEM ( $n=6$ /group). MOV: mechanical overloading.

chanical overloading. In addition, we also observed neonatal MHC isoforms in a small proportion of myotubes and/or myofibers during 4-7 days in the soleus muscle of mice after mechanical overloading. Therefore, the rodent soleus and plantaris muscles after mechanical overloading seem to receive damage to muscle fibers and undergo degeneration/regeneration. In fact, mechanical overloading induces wide-ranging inflammation involving infiltrating monocyte cells, and increased expression of various cytokines [26-28]. A break in the plasma membrane has been indicated to be essential for muscle hypertrophy, releasing chemical mediators (growth factors) of remodeling stored in the cell cytosol [e.g. fibroblast growth factor (FGF)] [24,25]. However, some classical research indicates no neo-formation of muscle fibers after mechanical overloading. Gollnick et al. [23] found no significant change in fiber numbers after mechanical overloading on directly counting individual fibers dissected from $\mathrm{HNO}_{3}$-treated muscles. Unfortunately, this laborious analysis could not determine whether degeneration/regeneration occur in the hypertrophic muscle after mechanical overloading, because it is possible that the numbers of newly formed fibers in hypertrophic muscles equal those in damaged and there 
Table 2. The percentage of muscle fibers prossessing Neonatal MHC and/or NFATc3 in the soleus muscle at 2 and 10 days after MOV.

\begin{tabular}{llllll}
\hline & Neo MHC $(+)$ & Neo MHC $(+)$ & total & Neo MHC $(-)$ & total \\
& NFATc3 $(-)$ & NFATc3 $(+)$ & Neo MHC & NFATc3 $(+)$ & NFATc3 \\
\hline Placebo 2d & $1.3 \pm 0.2$ & $0.1 \pm 0.1$ & $1.4 \pm 0.2$ & $1.5 \pm 0.8$ & $1.5 \pm 0.8$ \\
CsA 2d & $1.8 \pm 1.4$ & $0.4 \pm 0.1$ & $2.2 \pm 1.5$ & $1.1 \pm 1.0$ & $1.5 \pm 1.1$ \\
Placebo 10d & $0.5 \pm 0.4$ & $0.3 \pm 0.2$ & $0.8 \pm 0.4$ & $2.2 \pm 1.3$ & $2.5 \pm 1.4$ \\
CsA 10d & $0.1 \pm 0.1$ & $0.3 \pm 0.1$ & $0.4 \pm 0.2$ & $2.9 \pm 0.8$ & $3.2 \pm 0.9$ \\
\hline
\end{tabular}
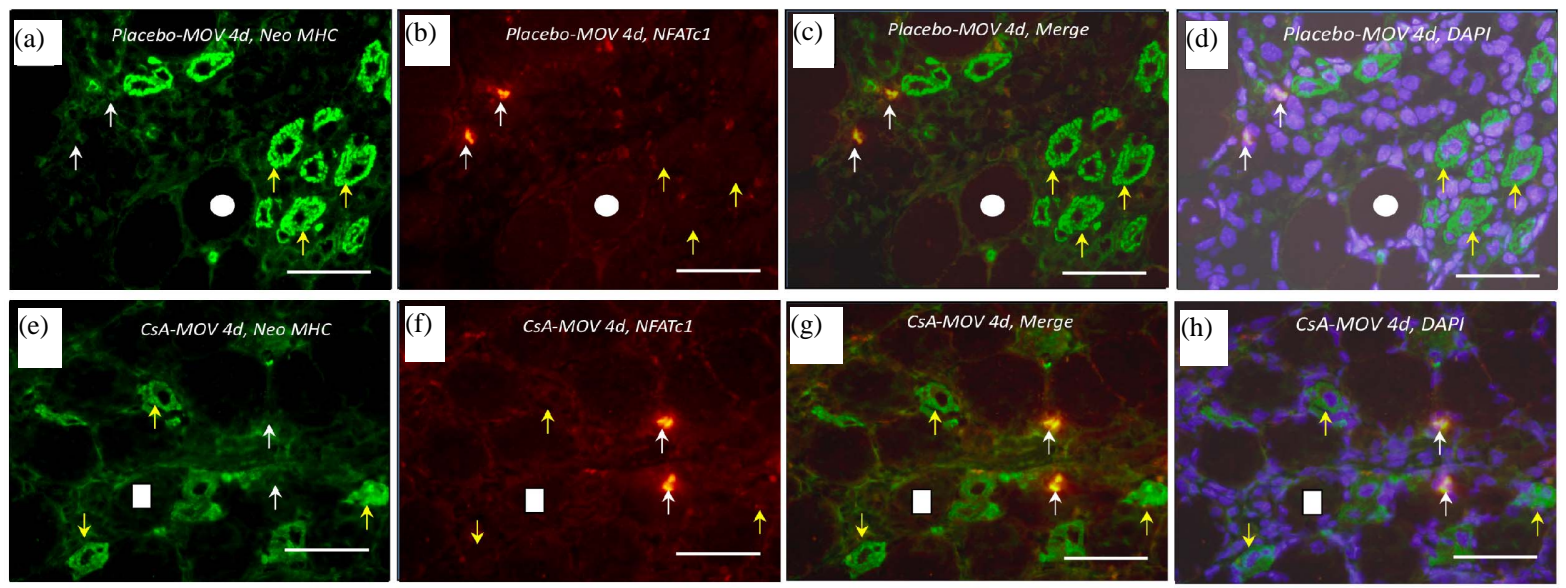

Figure 5. NFATc1 immunoreactivity was observed in some mononuclear cells in placebo-treated (b) and CsA- treated (f) mechanically overloaded muscles. The immunolocalization of NFATc1 did not correspond that in neonatal MHC-positive myotubes at 4 ((a)-(h)) and 7 days (data not shown). White circles and squares indicate the same fibers on different immunoimages. Whire arrows denote the mononuclear cells possessing NFATc1 but not neonatal MHC. Yellow allows denote the myotubes possessing neonatal MHC but not NFATc1. Bar $=50 \mu \mathrm{m}$.

after necrotic/apoptotic fibers.

We recognized the appearance of the neonatal MHC isoforms in a high proportion of soleus muscle fibers in both groups after 4 and 7 days of overload ( 20\% compared with $\sim 0.1 \%$ in either control group). These immature fibers could reflect the reexpression of this developmental isoform in existing mature fibers or the presence of new fibers. On the basis of the various sizes and the gradual growth with days of the myofibers containing neonatal MHC, it seems that the majority of these fibers are newly formed. Phelan and Gonyea [21] also recognized, in rodent hypertrophic soleus muscle after synergistic ablation, several populations (7\%) of embryonic MHC-positive myofibers. In addition, they suggested that these immature fibers are newly formed through satellite cell fusion because of the almost complete abolition of small fibers in irradiated muscle. Furthermore, embryonic MHC mRNA and protein have been detected in overloaded rat hindlimb [22,33] and in stretch-overloaded chicken ALD muscles [34].

The process by which satellite cells form myotubes is regulated by IGF-I-calcineurin signaling similar to myogenesis (myogenic differentiation). The downstream regulators of calcineurin are the four NFATs as well as the MEF2 family [13,35]. NFATc1 and NFATc3 promote the myogenic differentiation in vitro $[30,36]$. More recent findings [37] demonstrated the nucleocytoplasmic shuttling, after electrical stimulation, of all 4 NFAT isoforms in the EDL muscle of rats. These results suggest that NFATc1 and/or NFATc3, by stimulating calcineurin, enhance the growth of immature fibers in the soleus muscle after mechanical overloading. However, in the active differentiation phase (4 days), there was no change in the co-localization of neonatal MHC and NFATc3 in the overloaded soleus muscle after calcineurin inhibition (Figure 1). Treatment with CsA did not affect the percentage of fibers expressing NFATc3 in the muscle. In addition, the expression pattern of GSK3 $\beta$, a major regulator of NFATc3 signaling [31], was not altered in the soleus muscle by CsA treatment. Furthermore, the localization of NFATc1 did not correspond to that in neonatal MHC-positive myotubes (Figure 5), although NFATc1-immunoreactivity was observed in some mononuclear cells. Therefore, NFATc1 and NFATc3 may not help to regulate the expression of neonatal MHC. 
Other NFAT family members may regulate the hypertrophic process in the soleus muscle. Calabria et al. [37] found using RNA interference that all of the NFAT family regulate the expression of slow MHC in the regenerating soleus muscle of mice. Although NFATc4 seems to activate the mRNA expression of all forms of MHC (slow, MHC2A, MHC2X, and MHC2B), it regulates MHC2B mRNA expression differently to the other NFAT isoforms. Indeed, the amount of NFATc4 mRNA is reported to be significantly lower than that of any other NFAT in the slow-type soleus muscle. NFATc4 does not seem to regulate the hypertrophy of slow-twitch skeletal muscles. In contrast, mice with a null mutation of NFATc2 showed smaller type I as well as type II fibers in the soleus muscle [38]. In addition, growthretardation after muscle damage was recognized in the muscle of NFATc2-/- mice [38]. Thus, further study will be required to investigate the functional role of NFATc2 in the hypertrophied soleus muscle.

Some controversy exists as to whether all NFAT isoforms are expressed in skeletal muscle. Although the presence of all 4 NFATs has been shown at the RNA [39] and protein [40] level, some studies could not detect the expression of NFATc3 and c4 [9,41]. More recent findings [37] also indicated the expression of all 4 NFATs in adult skeletal muscle of the rat. The present study did not detect NFATc3 in control non-operated soleus muscle of mice. In non-operated soleus and EDL muscle of rats, immunolabeling of NFATc1 and NFATc4 was detected in myonucleus and/or satellite cells. Although this study indicated the presence of NFATc2 and NFATc3 in the myonucleus in control muscle, microphotographs did not show the existence of these proteins. Therefore, it may be difficult to show the cellular distribution of NFATc2 and NFATc3 in non-stimulated muscle as reflecting mRNA abundance.

The results presented in this paper highlight the complexity of calcineurin signaling during the hypertrophy of anti-gravity/postural soleus muscle in vivo. Calcineurin may regulate the expression of Neonatal MHC independent of NFATc1- and NFATc3 -dependent pathways. More work will be required to dissect each of the NFAT pathways to define the mechanisms and substrates of calcineurin in the overloaded soleus muscle.

\section{ACKNOWLEDGEMENTS}

This work was supported by a research Grant-in-Aid for Scientific Research C (No. 20500575) from the Ministry of Education, Science, Culture, Sports, Science and Technology of Japan.

\section{REFERENCES}

[1] Glass, D.J. (2003) Signalling pathways that mediate skeletal muscle hypertrophy and atrophy. Nature Cell Biology, 5, 87-90. doi:10.1038/ncb0203-87

[2] DeVol, D.L., Rotwein, P., Sadow, J.L., Novakofski, J. and Bechtel, P.J. (1990) Activation of insulin-like growth factor gene expression during work-induced skeletal muscle growth. American Journal of Physiology, 259, E89-E95.

[3] Sakuma, K., Watanabe, K., Totsuka, T., Uramoto, I., Sakamoto, K. and Sano, M. (1998) Differential adaptations of insulin-like growth factor, basic fibroblast growth factor and leukemia inhibitory factor in the plantaris muscle of rats by mechanical overloading: An immunohistochemical study. Acta Neuropathologica (Berl), 95, 123-130. doi:10.1007/s004010050775

[4] Coleman, M.E., DeMayo, F., Yin, K.C., Lee, H.M., Geske, R., Montgomery, C. and Schwartz, R.J. (1995) Myogenic vector expression of insulin-like growth factor I stimulates muscle cell differentiation and myofiber hypertrophy in transgenic mice. Journal of Biological Chemistry, 270, 12109-12116. doi:10.1074/jbc.270.20.12109

[5] Musaró, A., McCullagh, K., Paul, A., Houghton, L., Dobrowolny, G., Molinaro, M., Barton, E.R., Sweeney, H.L. and Rosenthal, N. (2001) Localized Igf-1 transgene expression sustains hypertrophy and regeneration in senescent skeletal muscle. Nature Genetics, 27, 195-200. doi:10.1038/84839

[6] Bodine, S.C., Stitt, T.N., Gonzalez, M., Kline, W.O., Stover, G.L., Bauerlein, R., Zlotchenko, E., Scrimgeour, A., Lawrence, J.C., Glass, D.J. and Yancopoulos, G.D. (2001) Akt/mTOR pathway is a crucial regulator of skeletal muscle hypertrophy and can prevent muscle atrophy in vivo. Nature Cell Biology, 3, 1014-1019. doi:10.1038/ncb1101-1014

[7] Lai, K.-M., Gonzalez, M., Poueymirou, W.T., Kline, W. O., Na, E., Zlotchenko, E., Stitt, T.N., Economides, A.N., Yancopoulos, G.D. and Glass, D.J. (2004) Conditional activation of akt in adult skeletal muscle induces rapid hypertrophy. Molecular and Cellular Biology, 24, 9295-9304. doi:10.1128/MCB.24.21.9295-9304.2004

[8] Dunn, S.E., Chin, E.R. and Michel, R.N. (2000) Matching of calcineurin activity to upstream effectors is critical for skeletal muscle fiber growth. Journal of Cell Biology, 151, 663-672. doi:10.1083/jcb.151.3.663

[9] Abbott, K.L., Friday, B.B., Thaloor, D., Murphy, T.J. and Pavlath, G.K. (1998) Activation and cellular localization of the cyclosporine A-sensitive transcription factor NF-AT in skeletal muscle cells. Molecular Biology of the Cell, 9, 2905-2916.

[10] Lara-Pezzi, E., Winn, N., Paul, A., McCullagh, K., Slominsky, E., Santini, M.P., Mourkioti, F., Sarathchandra, P., Fukushima, S., Suzuki, K. and Rosenthal, N. (2007) A naturally occurring calcineurin variant inhibits Foxo activity and enhances skeletal muscle regeneration. Journal of Cell Biology, 179, 1205-1218. doi:10.1083/jcb.200704179

[11] Sakuma, K., Nishikawa, J., Nakao, R., Watanabe, K., Totsuka, T., Nakano, H., Sano, M. and Yasuhara, M. (2003) Calcineurin is a potent regulator for skeletal muscle regeneration by association with NFATc1 and GATA-2. Acta Neuropathologica (Berl), 105, 271- 280.

[12] Sakuma, K., Nakao, R., Aoi, W., Inashima, S., Fujikawa, 
T., Hirata, M., Sano, M. and Yasuhara, M. (2005) Cyclosporin A treatment upregulates Id1 and Smad3 expression and delays skeletal muscle regeneration. Acta Neuropathologica (Berl), 110, 269-280. doi:10.1007/s00401-005-1049-x

[13] Sakuma, K. and Yamaguchi, A. (2010) The functional role of calcineurin in hypertrophy, regeneration, and disorders of skeletal muscle. Journal of Biomedicine and Biotechnology, Article ID: 721219. http://www.hindawi.com/journals/jbb/2010/721219/

[14] Chin, E.R., Olson, E.N., Richardson, J.A., Yang, Q., Humphries, C., Shelton, J.M., Wu, H., Zhu, W., Bassel-Duby, R. and Williams, R.S. (1998) A calcineurindependent transcriptional pathway controls skeletal muscle fiber type. Genes and Development, 12, 2499-2509. doi:10.1101/gad.12.16.2499

[15] Dunn, S.E., Simard, A.R., Bassel-Duby, R., Williams, R.S. and Michel, R.N. (2001) Nerve activity-dependent modulation of calcineurin signaling in adult fast and slow skeletal muscle fibers. Journal of Biological Chemistry, 276, 45243-45254. doi:10.1074/jbc.M105445200

[16] Oh, M., Rybkin, I.I., Copeland, V., Czubryt, M.P., Shelton, J.M., van Rooij, E., Richardson, J.A., Hill, J. A., De Windt, L.J., Bassel-Duby, R., Olson, E.N. and Rothermel, B.A. (2005) Calcineurin is necessary for the maintenance but not embryonic development of slow muscle fibers. Molecular and Cellular Biology, 25, 6629-6638. doi:10.1128/MCB.25.15.6629-6638.2005

[17] Naya, F.J., Mercer, B., Shelton, J., Richardson, J.A., Williams, R.S. and Olson, E.N. (2000) Stimulation of slow skeletal muscle fiber gene expression by calcineurin in vivo. Journal of Biological Chemistry, 275, 4545-4548. doi:10.1074/jbc.275.7.4545

[18] Sakuma, K., Akiho, M., Nakashima, H., Nakao, R., Hirata, M., Inashima, S., Yamaguchi, A. and Yasuhara, M. (2008) Cyclosporin A modulates cellular localization of MEF2C protein and blocks fiber hypertrophy in the overloaded soleus muscle of mice. Acta Neuropathologica (Berl), 115, 663-674.

[19] Talmadge, R.J., Otis, J.S., Rittler, M.R., Garcia, N.D., Spencer, S.R., Lees, S.J. and Naya F.J. (2004) Calcineurin activation influences muscle phenotype in a muscle-specific fashion. BMC Cell Biology, 5, Article 28. doi:10.1186/1471-2121-5-28

[20] Dunn, S.E. and Michel, R.N. (1997) Coordinated expression of myosin heavy chain isoforms and metabolic enzymes within overloaded rat muscle fibers. American Journal of Physiology, 273, C371-C383.

[21] Phelan, J.N. and Gonyea, W.J. (1997) Effect of radiation on satellite cell activity and protein expression in over loaded mammalian skeletal muscle. Anatomical Record, 247, 179-188.

doi:10.1002/(SICI)1097-0185(199702)247:2<179::AIDAR4>3.0.CO;2-T

[22] Roy, R.R., Talmadge, R.J., Fox, K., Lee, M., Ishihara, A. and Edgerton, V.R. (1997) Modulation of MHC isoforms in functionally overloaded and exercised rat plantaris fibers. Journal of Applied Physiology, 83, 280-290.

[23] Gollnick, P.D., Timson, B.F., Moore, R.L. and Riedy, M. (1981) Muscular enlargement and number of fibers in skeletal muscle of rats. Journal of Applied Physiology, 50, 936-943.
[24] Clarke, M.S.F., Caldwell, R.W., Miyake, K. and McNeil, P.L. (1995) Contraction-induced cell wounding and release of fibroblast growth factor in heart. Circulation Research, 76, 927-934.

[25] McNeil, P.L. and Steinhardt, R.A. (2003) Plasma membrane disruption: Repair, prevention, adaptation. Annual Review of Cell and Developmental Biology, 19, 697-731. doi:10.1146/annurev.cellbio.19.111301.140101

[26] Akiho, M., Nakashima, H., Sakata, M., Yamasa, Y., Yamaguchi, A. and Sakuma, K. (2010) Expression profile of Notch-1 in mechanically overloaded plantaris muscle of mice. Life Sciences, 86, 59-65. doi:10.1016/j.lfs.2009.11.011

[27] Armstrong, R.B., Marum, P., Tullson, P. and Saubert, C.W., IV (1979) Acute hypertrophic response of skeletal muscle to removal of synergists. Journal of Applied Physiology, 46, 835-842.

[28] Thompson, R.W., McClung, J.M., Baltgalvis, K.A., Davis, J.M. and Carson, J.A. (2006) Modulation of overload-induced inflammation by aging and anabolic steroid administration. Experimental Gerontology, 41, 1136-1148. doi:10.1016/j.exger.2006.08.013

[29] Musaró, A., McCullagh, K.J.A., Naya, F.J., Olson, E.N. and Rosenthal, N. (1999) IGF-I induces skeletal myocyte hypertrophy through calcineurin in association with GATA-2 and NF-ATc1. Nature, 400, 581-585. doi:10.1038/23060

[30] Delling, U., Tureckova, J., Lim, H.W., De Windt, L.J., Rotwein, P. and Molkentin, J.D. (2000) A calcineurinNFATc3-dependent pathway regulates skeletal muscle differentiation and slow myosin heavy-chain expression. Molecular and Cellular Biology, 20, 6600-6611. doi:10.1128/MCB.20.17.6600-6611.2000

[31] Van der Velden, J.L.J., Schols, A.M.W.J., Willems, J., Kelders, M.C.J.M. and Langen, R.C.J. (2008) Glycogen synthase kinase $3 \beta$ suppresses myogenin differentiation through negative regulation of NFATc3. Journal of Biological Chemistry, 283, 358-366. doi:10.1074/jbc.M707812200

[32] Michel, R.N., Dunn, S.E. and Chin, E.R. (2004) Calcineurin and skeletal muscle growth. Proceedings of the Nutrition Society, 63, 341-349. doi:10.1079/PNS2004362

[33] Periasamy, M., Gregory, P., Martin, B.J. and Stirewalt, W.S. (1989) Regulation of myosin heavy-chain gene expression during skeletal-muscle hypertrophy. Biochemical Journal, 257, 691-698.

[34] McCormick, K.M. and Schultz, E. (1992) Mechanisms of nascent fiber formation during avian skeletal muscle hypertrophy. Developmental Biology, 150, 319-334. doi:10.1016/0012-1606(92)90245-C

[35] Al-Shanti, N. and Stewart, C.E. (2009) Ca2+/calmodulindependent transcriptional pathways: Potential mediators of skeletal muscle growth and development. Biological Reviews Cambridge Phylosophical Society, 84, 637-652. doi:10.1111/j.1469-185X.2009.00090.x

[36] Semsarian, C., Wu, M.-J., Ju, Y.-K., Marciniec, T., Yeoh, T., Allen, D.G., Harvey, R.P. and Graham, R.M. (1999) Skeletal muscle hypertrophy is mediated by a Ca2+-dependent calcineurin signalling pathway. Nature, 400, 576-581. doi:10.1038/23054

[37] Calabria, E., Ciciliot, S., Moretti, I., Garcia, M., Picard, 
A., Dyar, K.A., Pallafacchina, G., Tothova, J., Schiaffino, S. and Murgia, M. (2009) NFAT isoforms control activity-dependent muscle fiber type specification. Proceedings of the National Academy of Sciences of the United States of America, 106, 13335-13340. doi:10.1073/pnas.0812911106

[38] Horsley, V., Friday, B.B., Matteson, S., Kegley, K.M., Gephart, J. and Pavlath, G.K. (2001) Regulation of the growth of multinucleated muscle cells by an NFATc2dependent pathway. Journal of Cell Biology, 153, 329-338. doi:10.1083/jcb.153.2.329

[39] Kegley, K.M., Gephart, J., Warren, G.L. and Pavlath, G. K. (2001) Altered primary myogenesis in NFATc3-/- mice leads to decreased muscle size in the adult. Developmental Biology, 232, 115-126.

doi:10.1006/dbio.2001.0179

[40] Parsons, S.A., Wilkins, B.J., Bueno, O.F. and Molkentin, J.D. (2003) Altered skeletal muscle phenotypes in calcineurin Aalpha and Abeta gene-targeted mice. Molecular and Cellular Biology, 23, 4331-4343. doi:10.1128/MCB.23.12.4331-4343.2003

[41] Swoap, S.J., Hunter, R.B., Stevenson, E.J. Felton, H.M., Kansagra, N.V., Lang, J.M., Esser, K.A. and Kandarian, S.C. (2000) The calcineurin-NFAT pathway and muscle fiber-type gene expression. American Journal of Physiology, 279, C915-C924. 